\title{
Hyper generalized pseudo $Q$-symmetric semi-Riemannian manifolds
}

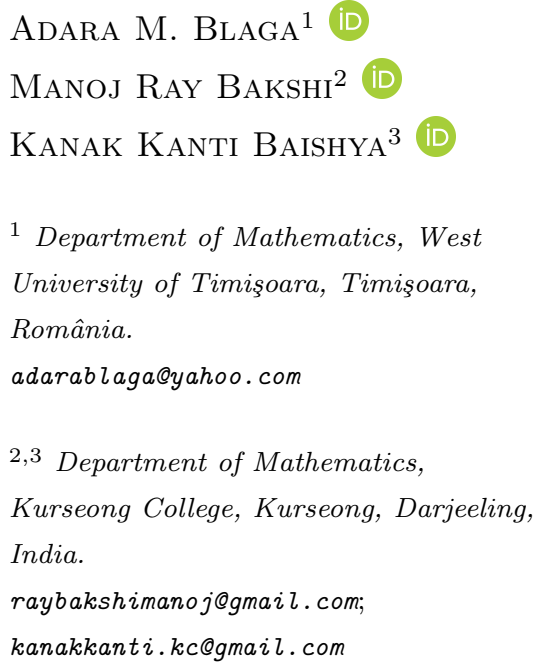

\section{ABSTRACT}

The object of the present paper is to study the properties of a hyper generalized pseudo $Q$-symmetric semi-Riemannian manifold, proving that under certain assumptions, it is a perfect fluid spacetime.

\section{RESUMEN}

El objetivo del presente artículo es estudiar las propiedades de una variedad semi-Riemanniana hiper generalizada pseudo $Q$-simétrica, probando que bajo ciertas condiciones, es un espacio-tiempo fluido perfecto.

Keywords and Phrases: $Q$-curvature tensor, perfect fluid spacetime.

2020 AMS Mathematics Subject Classification: 53C15, 53C25.

\section{(cc) BY-NC}




\section{Introduction}

Let $R, S, L$ and $r$ denote the curvature tensor, Ricci tensor, Ricci operator and the scalar curvature of a (semi)-Riemannian manifold, respectively. It is Mantica and Suh [5] who have introduced the notion of $Q$-curvature tensor. In an $n$-dimensional Riemannian or semi-Riemannian manifold $\left(M^{n}, g\right)(n>2)$, the $Q$-curvature tensor is defined as

$$
R(Y, U, V, W)=Q(Y, U, V, W)+\frac{\psi}{n-1}[g(Y, W) g(U, V)-g(Y, V) g(U, W)],
$$

where $Y, U, V, W$ are arbitrary vector fields on $M^{n}$ and $\psi$ is a scalar function. Semi-Riemannian manifolds with Ricci tensor $S$ of the form

$$
S(Y, V)=a g(Y, V)+b T(Y) T(V)
$$

for any vector fields $Y, V$, are often termed as perfect fluid spacetimes, where $a$ and $b$ are scalars and the vector field $\varrho$, metrically equivalent to the 1 -form $T$ (that is, $g(Y, \varrho)=T(Y)$ ), is a unit time like vector field (that is, $g(\varrho, \varrho)=-1$ ).

An $n$-dimensional semi-Riemannian manifold is said to be hyper generalized pseudo $Q$-symmetric (which will be abbreviated hereafter as $(H G P Q S)_{n}$ ) if it satisfies the equation

$$
\begin{aligned}
& \left(\nabla_{X} Q\right)(Y, U, V, W) \\
= & 2 A_{1}(X) Q(Y, U, V, W)+A_{1}(Y) Q(X, U, V, W) \\
& +A_{1}(U) Q(Y, X, V, W)+A_{1}(V) Q(Y, U, X, W) \\
& +A_{1}(W) Q(Y, U, V, X)+2 A_{2}(X)(g \wedge S)(Y, U, V, W) \\
& +A_{2}(Y)(g \wedge S)(X, U, V, W)+A_{2}(U)(g \wedge S)(Y, X, V, W) \\
& +A_{2}(V)(g \wedge S)(Y, U, X, W)+A_{2}(W)(g \wedge S)(Y, U, V, X),
\end{aligned}
$$

where

$$
\begin{aligned}
(g \wedge S)(Y, U, V, W)= & g(Y, W) S(U, V)+g(U, V) S(Y, W) \\
& -g(Y, V) S(U, W)-g(U, W) S(Y, V)
\end{aligned}
$$

and $A_{1}, A_{2}$ are non-zero 1 -forms whose $g$-dual vector fields will be denoted by $\theta_{1}$ and $\theta_{2}$, i.e. $A_{1}(X)=g\left(X, \theta_{1}\right)$ and $A_{2}(X)=g\left(X, \theta_{2}\right)$.

We organized our paper as follows: section 2 is concerned with preliminaries. After preliminaries, some curvature properties of $(H G P Q S)_{n}$ manifolds are studied in section 3 . It is obtained that the $Q$-curvature tensor in a $(H G P Q S)_{n}$ manifold satisfies 2nd Bianchi's identity. It is further obtained that the scalar function $\psi$ is always constant. In section 4 we investigate properties of divergence-free $(H G P Q S)_{n}$ manifolds and we prove that a divergence-free $(H G P Q S)_{n}$ manifold $(n>2)$ under the assumption $A_{1}(Q(Y, U) V)=0$ is a perfect fluid spacetime as well as the integral 
curves of the vector field $\varrho$ are geodesics and the vector field $\varrho$ is irrotational, if the associated vector fields $\varrho$ and $\sigma$ corresponding to the 1 -forms $T_{1}$ and $T_{2}$ are related by $(r-1) \varrho+n \sigma=0$.

\section{Preliminaries}

In this section, some relations useful to the study of $(H G P Q S)_{n}$ manifolds are obtained. Let $\left\{e_{i}\right\}$ be an orthonormal basis of the tangent space at each point of the manifold, where $1 \leq i \leq n$.

From (1.1) we can easily verify that the tensor $Q$ satisfies the following properties:

(i) $Q(Y, U) V+Q(U, Y) V=0$,

(ii) $Q(Y, U) V+Q(U, V) Y+Q(V, Y) U=0$,

where $g(Q(X, Y) U, V)=Q(X, Y, U, V)$.

Also from (1.1) we have

$$
\sum_{i=1}^{n} \epsilon_{i} Q\left(X, Y, e_{i}, e_{i}\right)=0=\sum_{i=1}^{n} \epsilon_{i} Q\left(e_{i}, e_{i}, W, U\right)
$$

and

$$
\begin{aligned}
\sum_{i=1}^{n} \epsilon_{i} Q\left(e_{i}, Y, V, e_{i}\right) & =\sum_{i=1}^{n} \epsilon_{i} Q\left(Y, e_{i}, e_{i}, V\right)=S(Y, V)-\psi g(Y, V) \\
& =: \quad Z(Y, V),
\end{aligned}
$$

where

$$
\epsilon_{i}=g\left(e_{i}, e_{i}\right)= \pm 1, S(X, Y)=\sum_{i=1}^{n} \epsilon_{i} g\left(R\left(X, e_{i}\right) e_{i}, Y\right), r=\sum_{i=1}^{n} \epsilon_{i} S\left(e_{i}, e_{i}\right)
$$

From (1.1) and (2.1) it follows that

(i) $Q(X, Y, U, V)+Q(X, Y, V, U)=0$,

(ii) $Q(X, Y, U, V)-Q(U, V, X, Y)=0$.

\section{Some curvature properties of $(H G P Q S)_{n}$ manifolds}

In this section we prove that in a $(H G P Q S)_{n}$ manifold, the $Q$-curvature tensor satisfies 2 nd Bianchi's identity, that is,

$$
\left(\nabla_{X} Q\right)(Y, U, V, W)+\left(\nabla_{Y} Q\right)(U, X, V, W)+\left(\nabla_{U} Q\right)(X, Y, V, W)=0
$$


In view of (1.1), (1.2) and (3.1) we get

$$
\begin{aligned}
& \left(\nabla_{X} Q\right)(Y, U, V, W)+\left(\nabla_{Y} Q\right)(U, X, V, W)+\left(\nabla_{U} Q\right)(X, Y, V, W) \\
= & A_{1}(V)[Q(Y, U, X, W)+Q(U, X, Y, W)+Q(X, Y, U, W)] \\
& +A_{1}(W)[Q(Y, U, V, X)+Q(U, X, V, Y)+Q(X, Y, V, U)] \\
& +A_{2}(V)[(g \wedge S)(Y, U, X, W)+(g \wedge S)(U, X, Y, W) \\
& +(g \wedge S)(X, Y, U, W)]+A_{2}(W)[(g \wedge S)(Y, U, V, X) \\
& +(g \wedge S)(U, X, V, Y)+(g \wedge S)(X, Y, V, U)] .
\end{aligned}
$$

Using (1.3) and 1st Bianchi's identity for the $Q$-curvature tensor in (3.2) and then simplifying, we obtain (3.1).

Thus we can state the following:

Theorem 3.1. The Q-curvature tensor in a $(H G P Q S)_{n}$ manifold satisfies 2nd Bianchi's identity.

Using (1.1) in (3.1), we have

$$
\begin{aligned}
\left(\nabla_{X} R\right)(Y, U, V, W) & +\left(\nabla_{Y} R\right)(U, X, V, W)+\left(\nabla_{U} R\right)(X, Y, V, W) \\
& -\frac{d \psi(X)}{(n-1)}[g(Y, W) g(U, V)-g(Y, V) g(U, W)] \\
& -\frac{d \psi(Y)}{(n-1)}[g(U, W) g(X, V)-g(U, V) g(X, W)] \\
& -\frac{d \psi(U)}{(n-1)}[g(X, W) g(Y, V)-g(X, V) g(Y, W)]=0 .
\end{aligned}
$$

By virtue of 2nd Bianchi's identity for the Riemannian curvature tensor, (3.3) yields

$$
\begin{aligned}
& \frac{d \psi(X)}{(n-1)}[g(Y, W) g(U, V)-g(Y, V) g(U, W)] \\
+ & \frac{d \psi(Y)}{(n-1)}[g(U, W) g(X, V)-g(U, V) g(X, W)] \\
+ & \frac{d \psi(U)}{(n-1)}[g(X, W) g(Y, V)-g(X, V) g(Y, W)]=0 .
\end{aligned}
$$

Contracting $U$ and $V$ in (3.4), we have

$$
(n-2)[d \psi(X) g(Y, W)-d \psi(Y) g(X, W)]=0
$$

which yields after further contraction

$$
(n-1)(n-2) d \psi(X)=0 .
$$

This implies that $d \psi(X)=0$, that is, $\psi$ is constant since $n>2$ and leads to the following:

Theorem 3.2. In a $(H G P Q S)_{n}$ manifold, the scalar function $\psi$ is always constant. 
Consequently, one can easily bring out the following:

Theorem 3.3. In a $(H G P Q S)_{n}$ manifold, $(\operatorname{div} Q)(X, Y) Z$ and $(\operatorname{div} R)(X, Y) Z$ are equivalent.

In view of (1.1), (1.2) and Theorem 3.2 we have

$$
\begin{aligned}
& \left(\nabla_{X} R\right)(Y, U, V, W) \\
= & 2 A_{1}(X) Q(Y, U, V, W)+A_{1}(Y) Q(X, U, V, W) \\
& +A_{1}(U) Q(Y, X, V, W)+A_{1}(V) Q(Y, U, X, W) \\
& +A_{1}(W) Q(Y, U, V, X)+2 A_{2}(X)(g \wedge S)(Y, U, V, W) \\
& +A_{2}(Y)(g \wedge S)(X, U, V, W)+A_{2}(U)(g \wedge S)(Y, X, V, W) \\
& +A_{2}(V)(g \wedge S)(Y, U, X, W)+A_{2}(W)(g \wedge S)(Y, U, V, X)
\end{aligned}
$$

which yields

$$
\begin{aligned}
& \left(\nabla_{X} S\right)(U, V) \\
=\quad & {\left[F_{1}(X)+F_{2}(X)\right] S(U, V)+F_{2}(U) S(X, V)+F_{2}(V) S(U, X) } \\
+ & {\left[F_{3}(X)+F_{4}(X)\right] g(U, V)+F_{4}(U) g(X, V)+F_{4}(V) g(U, X) } \\
+ & A_{1}(Q(X, U) V)-A_{1}(Q(V, X) U)
\end{aligned}
$$

after contraction over $Y$ and $W$, where

$$
\begin{aligned}
& F_{1}(X)=A_{1}(X)+(n+1) A_{2}(X), \\
& F_{2}(X)=A_{1}(X)+(n-3) A_{2}(X), \\
& F_{3}(X)=r A_{2}(X)-\psi A_{1}(X)+3 A_{2}(L X), \\
& F_{4}(X)=r A_{2}(X)-\psi A_{1}(X)-A_{2}(L X),
\end{aligned}
$$

where $L$ is the Ricci operator defined by $g(L X, Y)=S(X, Y)$.

Definition 3.4. An n-dimensional semi-Riemannian manifold is called almost generalized pseudo Ricci symmetric if the non-flat Ricci curvature tensor satisfies the equation

$$
\begin{aligned}
& \left(\nabla_{X} S\right)(U, V) \\
=\quad & {[A(X)+B(X)] S(U, V)+A(U) S(X, V)+A(V) S(U, X) } \\
+ & {[C(X)+D(X)] g(U, V)+C(U) g(X, V)+C(V) g(U, X), }
\end{aligned}
$$

where $A, B, C$ and $D$ are non-zero 1 -forms whose $g$-dual vector fields will be denoted by $\gamma_{1}, \gamma_{2}, \delta_{1}$ and $\delta_{2}$, i.e. $A(X)=g\left(X, \gamma_{1}\right), B(X)=g\left(X, \gamma_{2}\right), C(X)=g\left(X, \delta_{1}\right)$ and $D(X)=g\left(X, \delta_{2}\right)$.

Thus we can state the following: 
Theorem 3.5. A $(H G P Q S)_{n}$ manifold $(n>2)$ under the assumption $A_{1}(Q(X, U) V)$ $=A_{1}(Q(V, X) U)$ is necessarily almost generalized pseudo Ricci symmetric.

Making use of (2.3) in (3.7), we get

$$
\begin{aligned}
& \left(\nabla_{X} Z\right)(U, V) \\
=\quad & {\left[F_{1}(X)+F_{2}(X)\right] Z(U, V)+F_{2}(U) Z(X, V)+F_{2}(V) Z(U, X) } \\
+ & {\left[F_{3}(X)+\psi F_{1}(X)+F_{4}(X)+\psi F_{2}(X)\right] g(U, V) } \\
+ & {\left[F_{4}(U)+\psi F_{2}(U)\right] g(X, V)+\left[F_{4}(V)+\psi F_{2}(V)\right] g(U, X), }
\end{aligned}
$$

where $Z=S-\psi g$ is the tensor considered in ([4], [6], [7]). This leads to the following:

Theorem 3.6. $A \quad(H G P Q S)_{n}$ manifold $(n>2)$ under the assumption $A_{1}(Q(X, U) V)$ $=A_{1}(Q(V, X) U)$ is necessarily almost generalized pseudo Z-symmetric.

\section{$4 \quad(\mathbf{H G P Q S})_{n}$ manifolds $(n>2)$ with $\operatorname{div} \mathbf{Q}=\mathbf{0}$}

Let $\left(M^{n}, g\right)$ be a semi-Riemannian manifold of dimension $n$ and let $\left\{e_{i}\right\}$ be an orthonormal basis of the tangent space $T_{p} M$ at any point $p \in M$ and $\epsilon_{i}= \pm 1$. Then the divergence of a vector field $U$ is defined as

$$
\operatorname{div} U=\sum_{i=1}^{n} \epsilon_{i} g\left(\nabla_{e_{i}} U, e_{i}\right)
$$

and the divergence of a tensor field of type $(1,3)$, which is a tensor field of type $(0,3)$, is defined as

$$
(\operatorname{div} K)(X, Y) Z=\sum_{i=1}^{n} \epsilon_{i} g\left(\left(\nabla_{e_{i}} K\right)(X, Y) Z, e_{i}\right)
$$

Now

$$
\begin{aligned}
(\operatorname{div} Q)(Y, U) V= & \sum_{i=1}^{n} \epsilon_{i} g\left(\left(\nabla_{e_{i}} Q\right)(Y, U) V, e_{i}\right) \\
= & \sum_{i=1}^{n} \epsilon_{i}\left[2 A_{1}\left(e_{i}\right) Q\left(Y, U, V, e_{i}\right)+A_{1}(Y) Q\left(e_{i}, U, V, e_{i}\right)\right. \\
& +A_{1}(U) Q\left(Y, e_{i}, V, e_{i}\right)+A_{1}(V) Q\left(Y, U, e_{i}, e_{i}\right) \\
& +A_{1}\left(e_{i}\right) Q\left(Y, U, V, e_{i}\right)+2 A_{2}\left(e_{i}\right)(g \wedge S)\left(Y, U, V, e_{i}\right) \\
& +A_{2}(Y)(g \wedge S)\left(e_{i}, U, V, e_{i}\right)+A_{2}(U)(g \wedge S)\left(Y, e_{i}, V, e_{i}\right) \\
& \left.+A_{2}(V)(g \wedge S)\left(Y, U, e_{i}, e_{i}\right)+A_{2}\left(e_{i}\right)(g \wedge S)\left(Y, U, V, e_{i}\right)\right]
\end{aligned}
$$




$$
\begin{aligned}
= & 3 A_{1}(Q(Y, U) V)+A_{1}(Y)[S(U, V)-\psi g(U, V)] \\
& -A_{1}(U)[S(Y, V)-\psi g(Y, V)]+3 A_{2}(Y) S(U, V) \\
& +3 A_{2}(L Y) g(U, V)-3 A_{2}(L U) g(Y, V)-3 A_{2}(U) S(Y, V) \\
& +A_{2}(Y)[(n-2) S(U, V)+r g(U, V)] \\
& -A_{2}(U)[(n-2) S(Y, V)+r g(Y, V)] \\
= & 3 A_{1}(Q(Y, U) V)+S(U, V)\left[A_{1}(Y)+(n+1) A_{2}(Y)\right] \\
& -S(Y, V)\left[A_{1}(U)+(n+1) A_{2}(U)\right] \\
& +g(U, V)\left[3 A_{2}(L Y)+r A_{2}(Y)-\psi A_{1}(Y)\right] \\
& -g(Y, V)\left[3 A_{2}(L U)+r A_{2}(U)-\psi A_{1}(U)\right] \\
= & 3 A_{1}(Q(Y, U) V)+T_{1}(Y) S(U, V)-T_{1}(U) S(Y, V) \\
& +T_{2}(Y) g(U, V)-T_{2}(U) g(Y, V),
\end{aligned}
$$

hence

$$
\begin{aligned}
(\operatorname{div} Q)(Y, U) V= & 3 A_{1}(Q(Y, U) V)+T_{1}(Y) S(U, V)-T_{1}(U) S(Y, V) \\
& +T_{2}(Y) g(U, V)-T_{2}(U) g(Y, V),
\end{aligned}
$$

where

$$
\begin{aligned}
& T_{1}(Y)=A_{1}(Y)+(n+1) A_{2}(Y)=: g(Y, \varrho), \text { for } \varrho=\theta_{1}+(n+1) \theta_{2}, \\
& T_{2}(Y)=3 A_{2}(L Y)+r A_{2}(Y)-\psi A_{1}(Y)=: g(Y, \sigma), \text { for } \sigma=3 L \theta_{2}+r \theta_{2}-\psi \theta_{1} .
\end{aligned}
$$

Assuming $(\operatorname{div} Q)(Y, U) V=0$ and $A_{1}(Q(Y, U) V)=0$, we get from the above equation

$$
T_{1}(Y) S(U, V)+T_{2}(Y) g(U, V)=T_{1}(U) S(Y, V)+T_{2}(U) g(Y, V) .
$$

Now contracting (4.2) over $U$ and $V$ we get

$$
S(Y, \varrho)=r T_{1}(Y)+(n-1) T_{2}(Y) .
$$

Again putting $V=\varrho$ in $(4.2)$ we get

$$
(n-2)\left[T_{1}(Y) T_{2}(U)-T_{1}(U) T_{2}(Y)\right]=0,
$$

which under the assumption $n>2$ implies $T_{1}(Y) T_{2}(U)=T_{1}(U) T_{2}(Y)$.

Now putting $U=\varrho$ in (4.2) and using (4.3) and (4.4) we get

$$
T_{1}(\varrho) S(Y, V)+T_{2}(\varrho) g(Y, V)=T_{1}(Y)\left[r T_{1}(V)+n T_{2}(V)\right]
$$

and we can state: 
Theorem 4.1. A divergence-free $(H G P Q S)_{n}$ manifold $(n>2)$ under the assumption $A_{1}(Q(Y, U) V)=0$ is a perfect fluid spacetime with unit timelike vector field $\varrho$, provided the associated vector fields $\varrho$ and $\sigma$ corresponding to the 1 -forms $T_{1}$ and $T_{2}$ are related by $(r-1) \varrho+n \sigma=0$.

In this case, (4.5) becomes

$$
S(Y, V)=a g(Y, V)-T_{1}(Y) T_{1}(V)
$$

where $a=: T_{2}(\varrho)$.

Again, $(\operatorname{div} Q)(Y, U) V=0$ gives

$$
\left(\nabla_{Y} S\right)(U, V)-\left(\nabla_{U} S\right)(Y, V)=0
$$

Now using (4.6) in (4.7) we find

$$
\begin{aligned}
& d a(Y) g(U, V)-d a(U) g(Y, V) \\
& -\left[T_{1}(V)\left(\nabla_{Y} T_{1}\right)(U)+T_{1}(U)\left(\nabla_{Y} T_{1}\right)(V)\right] \\
& +\left[T_{1}(V)\left(\nabla_{U} T_{1}\right)(Y)+T_{1}(Y)\left(\nabla_{U} T_{1}\right)(V)\right]=0 .
\end{aligned}
$$

Taking a frame field and contracting $Y$ and $V$ we get

$$
(n-1) d a(U)+\left[T_{1}(U)\left(\delta T_{1}\right)+\left(\nabla_{\varrho} T_{1}\right)(U)\right]=0,
$$

where

$$
\delta T_{1}=\sum_{i=1}^{n} \epsilon_{i}\left(\nabla_{e_{i}} T_{1}\right)\left(e_{i}\right) .
$$

Setting $V=Y=\varrho$ in (4.8) we find

$$
\left(\nabla_{\varrho} T_{1}\right)(U)=-d a(U)-d a(\varrho) T_{1}(U)
$$

Substituting (4.10) in (4.9) we get

$$
(n-2) d a(U)+T_{1}(U)\left(\delta T_{1}\right)-d a(\varrho) T_{1}(U)=0
$$

which yields

$$
\delta T_{1}=(n-1) d a(\varrho)
$$

for $U=\varrho$.

Using (4.12) in (4.11) we obtain

$$
d a(U)=-T_{1}(U) d a(\varrho),
$$

provided $n>2$. 
Putting $V=\varrho$ in (4.8) and using (4.13) we get

$$
\left(\nabla_{Y} T_{1}\right)(U)-\left(\nabla_{U} T_{1}\right)(Y)=0
$$

This means that the 1 -form $T_{1}$ is closed, that is,

$$
d T_{1}(Y, U)=0 .
$$

Hence

$$
g\left(\nabla_{U} \varrho, Y\right)=g\left(\nabla_{Y} \varrho, U\right) \text { for all } U, Y,
$$

which yields

$$
g\left(\nabla_{\varrho} \varrho, Y\right)=g\left(\nabla_{Y} \varrho, \varrho\right),
$$

for $U=\varrho$. Since $g\left(\nabla_{Y} \varrho, \varrho\right)=0$, from (4.15) it follows that $g\left(\nabla_{\varrho} \varrho, Y\right)=0$ for all $Y$. Hence $\nabla_{\varrho} \varrho=0$. This implies that the integral curves of the vector field $\varrho$ are geodesics. Therefore we can state the following:

Theorem 4.2. In a divergence-free $(H G P Q S)_{n}$ manifold $(n>2)$ under the assumption $A_{1}(Q(Y, U) V)=0$, the integral curves of the unit timelike vector field $\varrho$ are geodesics, provided the associated vector fields $\varrho$ and $\sigma$ corresponding to the 1 -forms $T_{1}$ and $T_{2}$ are related by $(r-1) \varrho+n \sigma=0$.

Taking into account that the divergence of the conformal curvature tensor of a Riemannian manifold $\left(M^{n}, g\right)$ is $([3],[6])$ :

$$
\begin{aligned}
(\operatorname{div} C)(X, Y) Z & =\frac{n-3}{n-2}\left[\left(\nabla_{X} S\right)(Y, Z)-\left(\nabla_{Y} S\right)(X, Z)\right] \\
& =\frac{n-3}{n-2}(\operatorname{div} Q)(X, Y) Z
\end{aligned}
$$

for any vector fields $X, Y, Z$ on $M^{n}$, from the Lemma 2.1 of [2] we infer

Theorem 4.3. Let $(M, g)$ be a $(H G P Q S)_{n}$ perfect fluid spacetime $(n>2)$. If $(\operatorname{div} Q)(X, Y) Z=0$, for any vector fields $X, Y, Z$ on $M$, then the unit timelike vector field $\varrho$ is irrotational.

Also, in [2] was proved the following result:

Theorem 4.4. [2] Let $(M, g)$ be a $(H G P Q S)_{n}$ perfect fluid spacetime $(n>2)$. If $(\operatorname{div} Q)(X, Y) Z=$ 0 , for any vector fields $X, Y, Z$ on $M$, then $(M, g)$ is a $G R W$ spacetime whose fiber is Einstein.

Acknowledgements. The authors are grateful to the referees for the valuable suggestions and remarks that definitely improved the paper. 


\section{References}

[1] K. K. Baishya, F. Ozen Zengin and J. Mikeš, "On hyper generalised weakly symmetric manifolds", Nineteenth International Conference on Geometry, Integrability and Quantization, 02 07, June 2017, Varna, Bulgaria Ivaïlo M. Mladenov and Akira Yoshioka, Editors Avangard Prima, Sofia 2018, pp. 1-10.

[2] C. A. Mantica, U. C. De, Y. J. Suh, and L. G. Molinari, "Perfect fluid spacetimes with harmonic generalized curvature tensor", Osaka J. Math., vol. 56, pp. 173-182, 2019.

[3] C. A. Mantica, and L. G. Molinari, "A second-order identity for the Riemann tensor and applications", Colloq. Math., vol. 122, no. 1, pp. 69-82, 2011.

[4] C. A. Mantica, and L. G. Molinari, "Weakly Z-symmetric manifolds", Acta Math. Hung., vol. 135, no. 1-2, pp. 80-96, 2012.

[5] C. A. Mantica, and Y. J. Suh, "Pseudo Q-symmetric semi-Riemannian manifolds", Int. J. Geom. Meth. Mod. Phys., vol. 10, no. 5, 2013.

[6] C. A. Mantica, and Y. J. Suh, "Pseudo Z-symmetric Riemannian manifolds with harmonic curvature tensors", Int. J. Geom. Meth. Mod. Phys., vol. 9, no. 1, 2012, 1250004.

[7] C. A. Mantica, and Y. J. Suh, "Recurrent Z-forms on Riemannian and Kaehler manifolds", Int. J. Geom. Meth. Mod. Phys., vol. 9, no. 7, 2012, 1250059. 